\title{
e Neuromuscular Ultrasound in Carpal Tunnel Syndrome: A Tool for Cutting a Gordian Knot
}

\section{TO THE EDITOR}

I read with great interest the observational study from Kim et al (1) regarding the relative location of the median nerve and ulnar artery to the palmaris longus (PL) tendon around the wrist in carpal tunnel syndrome (CTS). This observational study succeeded not only in highlighting interesting anatomical aspects of the wrist, but also depicted, that real time ultrasoundguided local steroid injection could be used as a safe and accurate technique for carpal tunnel syndrome therapy.

I would like to present the preliminary results from an observational study concerning the clinical significance of the distance between the median nerve and ulnar neurovascular bundle (MN-UA) and the critical role of neuromuscular ultrasound in choosing the appropriate therapy for CTS patients. In our ultrasound lab we perform pre- and postoperative high frequency ultrasound $(18 \mathrm{Mhz})$ of carpal tunnel in patients with clinical and electrophysiological signs of CTS. Fifteen patients (mean age 53.5y, SD $\pm 5.6,3$ women) with known CTS were enrolled into the study. The cross sectional area (CSA) of the median (at the level of retinaculum flexorum) and ulnar nerve (at the level of Guyon's canal) and the MN-UA were measured in all patients in the pre- and postoperative phase and were compared with the reference values of our lab $(2,3)$. Five patients underwent an open carpal tunnel release (O-CTR) surgery, 5 an endoscopic carpal tunnel release (E-CTR) and 5 received an ultrasound guided steroid injection, using the ulnar approach.

Table 1 shows the preoperative CSA and MN-UA measures. The postoperative ultrasound follow-up of the patients revealed a significant reduction of the CSA of the median nerve in both the O-CTR $(P=0.023)$ and E-CTR groups $(P=0.024)$, reflecting the clinical improvement of the patients (Table 2). The group of patients, which underwent ultrasound guided steroid injection, showed also clinical improvement, but no statistical significant alteration of the CSA of the median nerve could be found $(P=0.67)$. It is interesting, that in the E-CTR group a significant increase of the CSA of the ulnar nerve in Guyon's canal was documented $((P=$ 0.038) (Table 2).

Due to this ultrasound finding, all 5 patients of the endoscopic group underwent postoperative clinical and electrophysiological evaluation of the ulnar nerve. In 2 of the patients we documented clinical signs of ulnar neuropathy (Medical Research Council scale in

Table 1. Overview of the data from the preoperative ultrasound evaluation of the patients suffering from carpal tunnel syndrom

\begin{tabular}{|c|c|c|c|c|c|c|}
\hline & \multirow{2}{*}{ Nerve } & \multirow{2}{*}{ Location } & \multicolumn{2}{|c|}{ CSA } & \multicolumn{2}{|c|}{ MN-UA } \\
\hline & & & Mean & $\mathrm{SD}$ & Mean & SD \\
\hline \multirow{2}{*}{ O-CTR } & Median & Carpal Tunnel & 13.5 & 2.3 & 5.5 & 2.5 \\
\hline & Ulnar & Guyon's canal & 6.2 & 1.4 & & \\
\hline \multirow{2}{*}{ E-CTR } & Median & Carpal Tunnel & 14.8 & 2.2 & 5.2 & 2.3 \\
\hline & Ulnar & Guyon's canal & 5.7 & 1.8 & & \\
\hline \multirow{2}{*}{ US Guided Injection } & Median & Carpal Tunnel & 12.8 & 2.3 & 5.7 & 1.9 \\
\hline & Ulnar & Guyon's canal & 6.4 & 1.6 & & \\
\hline
\end{tabular}

Legend: O-CTR= open carpal tunnel release, E-CTR= endoscopic carpal tunnel release, $\mathrm{CSA}=$ cross sectional area, MN-UA: distance between the median nerve and ulnar neurovascular bundle, $\mathrm{US}=$ ultrasound, $\mathrm{SD}=$ standard deviation, $\mathrm{p}$ values $<0.05$ were considered as statistically significant and are highlighted with bold. 
Table 2. Overview of the data from the postoperative ultrasound evaluation of the patients, that underwent therapy for carpal tunnel syndrome.

\begin{tabular}{|c|c|c|c|c|c||}
\hline \hline \multirow{2}{*}{ Nerve } & \multirow{2}{*}{ Location } & \multicolumn{3}{|c|}{ CSA } \\
\cline { 3 - 6 } & & & Mean & SD & $P$ \\
\hline \multirow{2}{*}{ O-CTR } & Median & Carpal Tunnel & 10.2 & 1.3 & 0.023 \\
\cline { 2 - 6 } & Ulnar & Guyon's canal & 6.1 & 1.6 & 0.912 \\
\hline \multirow{2}{*}{ E-CTR } & Median & Carpal Tunnel & 11.2 & 1.9 & 0.024 \\
\cline { 2 - 6 } & Ulnar & Guyon's canal & 8.1 & 1.2 & 0.038 \\
\hline \multirow{2}{*}{ US Guided Injection } & Median & Carpal Tunnel & 12.2 & 2.1 & 0.67 \\
\cline { 2 - 7 } & Ulnar & Guyon's canal & 6.4 & 1.6 & 1 \\
\hline
\end{tabular}

Legend: $\mathrm{O}-\mathrm{CTR}=$ open carpal tunnel release, $\mathrm{E}-\mathrm{CTR}=$ endoscopic carpal tunnel release, $\mathrm{CSA}=$ cross sectional area, $\mathrm{MN}-\mathrm{UA}$ : distance between the median nerve and ulnar neurovascular bundle, $\mathrm{US}=$ ultrasound, $\mathrm{SD}=$ standard deviation, $\mathrm{p}$ values $<0.05$ were considered as statistically significant and are highlighted with bold.

Patient 1: abductor digiti minimi 4 and flexor digiti minimi 3; in Patient 2 abductor digiti minimi 3 and flexor digiti minimi 3). The electrophysiological examination confirmed the neuropathy in both patients (Patient 1: distal motor latency $5,6 \mathrm{~ms}$, compound muscle action potential $2,3 \mathrm{mV}$, motor conduction velocity of $23,2 \mathrm{~m} / \mathrm{sec}$, sensible nerve action potential of $2,1 \mu \mathrm{V}$ Patient 2: distal motor latency $6,1 \mathrm{~ms}$, compound muscle action potential $2,5 \mathrm{mV}$, motor conduction velocity of $24,1 \mathrm{~m} / \mathrm{sec}$, sensible nerve action potential of $2,3 \mu \mathrm{V}$ ). Interestingly both of the patients showed a distance between the median nerve and ulnar neurovascular bundle (MN-UA) of $3.0 \mathrm{~mm}$.

Considering this finding, we performed a review of the literature about this anatomic variation. Nakamichi et al. described a considerable variation of this distance among individuals (4). There is also strong evidence in the literature, that this anatomic variation is associated with an increased risk of intraoperative complications, such as injury of the ulnar neurovascular bundle, especially when performing an E-CTR $(4,5)$. A possible explanation for this risk could be, the proximity of the zone between

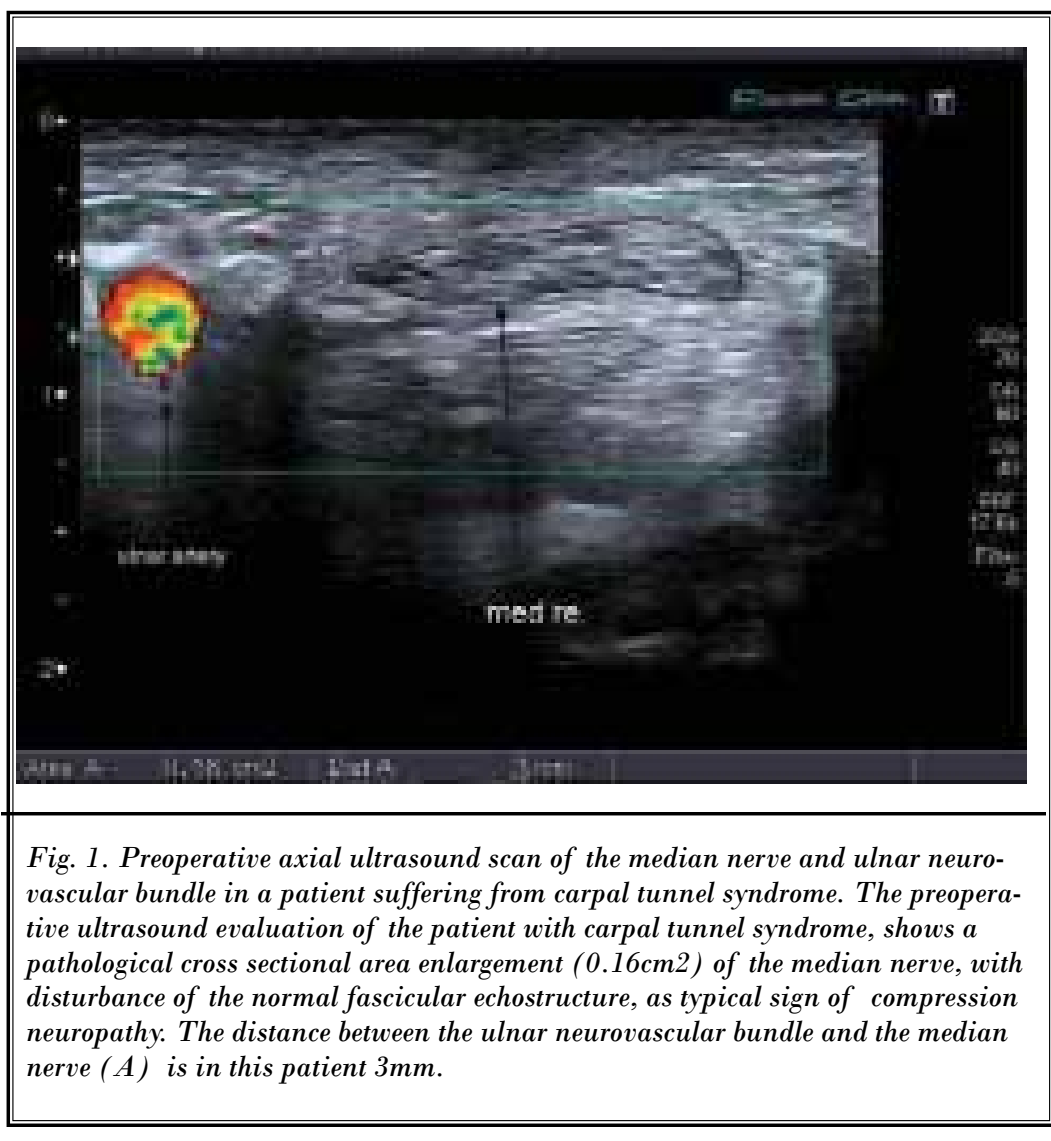

the median nerve and ulnar neurovascular bundle to the cutting device, as most endoscopic devices are designed to divide the flexor retinaculum just to the radial aspect of the hamate hook. According to a study of Cobb et al. (6) in cadaver specimens, the ulnar neurovascular bundle courses in the majority of patients radial to the hamate hook, being at high risk for injury, especially when endoscopic visualization is poor. 
Therefore, our preliminary findings highlight the critical role of neuromuscular ultrasound not only as diagnostic method of CTS, but also in choosing the appropriate therapy for CTS patients. Although the number of patients included in our observational study is limited, it seems that the preoperative ultrasound evaluation of the distance between the median nerve and ulnar neurovascular bundle (MN-UA) may help to identify patients being at risk for iatrogenic lesion.

Antonios Kerasnoudis, MD

Department of Neurology

Ruhr University

St. Josef-Hospital,

Gudrunstr. 56, 44791

Bochum, Germany

Email: antonis.kerasnoudis@gmail.co

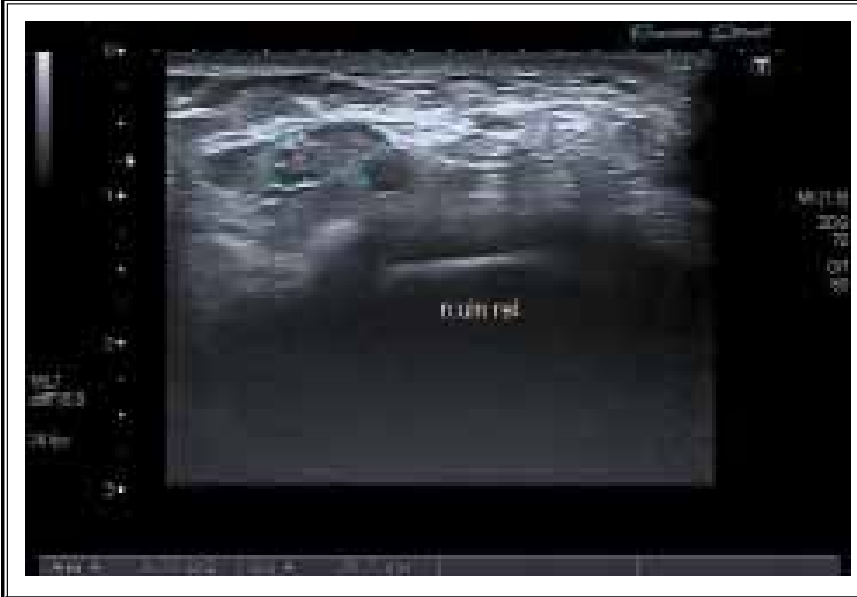

Fig. 2. Postperative ultrasound scan of the ulnar nerve in Guyon's canal in a patient, who underwent endoscopic carpal tunnel release for treatment of carpal tunnel syndrome. The postperative axial ultrasound scan of the ulnar nerve shows a pathological cross sectional area enlargement $(0.11 \mathrm{~cm} 2)$ of the ulnar nerve, with disturbance of the normal fascicular echostructure, as typical sign of neuropathy.

\section{References:}

1. Kim DH, Jang JE, Park BK. Anatomical basis of ulnar approach in carpal tunnel injection. Pain Physician 2013; 16:E191E198.

2. Kerasnoudis A, Pitarokoili K, Behrendt V, Gold R, Yoon MS. Cross sectional area reference values for sonography of peripheral nerves and brachial plexus. Clin Neurophysiol 2013; doi:pii: S1388-2457(13)00221-6. 10.1016/j. clinph.2013.03.007

3. Kerasnoudis A, Pitarokoili K. Ulnar nerve reference values for cross-sectional area, 5 . intranerve cross sectional area variability and side to side difference ratio. Rheumatol Int 2013 Feb 28. [Epub ahead of print]

4. Nakamichi K, Tachibana S. Distance between the median nerve and ulnar neurovascular bundle: clinical significance with ultrasonographically assisted carpal tunnel release. J Hand Surg Am 1998; 23:870-874.
Frick A, Baumeister RG, Kopp R. Choice of procedure in therapy of distal median nerve compression syndrome. Handchir Mikrochir Plast Chir 1996; 28:147-150.

6. Cobb TK, Carmichael SW, Cooney WP. The ulnar neurovascular bundle at the wrist. A technical note on endoscopic carpal tunnel release. J Hand Surg $\mathrm{Br}$ 1994; 19:24-26. 